\title{
Underwater communication using UAV to realize high-speed AUV deployment
}

\author{
Yusuke Yokota ${ }^{1, *}$ and Takumi Matsuda ${ }^{2}$ \\ 1 Institute of Industrial Science, University of Tokyo; yyokota@iis.u-tokyo.ac.jp \\ 2 Meiji University; tmatsuda@meiji.ac.jp \\ * Correspondence: yyokota@iis.u-tokyo.ac.jp
}

\begin{abstract}
To monitor the detail information in the sea and on the seafloor, sensors are generally installed on Autonomous Underwater Vehicle (AUV). AUV cannot accurately grasp the absolute position and needs to communicate with the sea-surface vehicle. However, the sea-surface vehicle cannot perform observation operations at high speed and with high efficiency due to low mobility and high human and financial costs. From this perspective, Unmanned Aerial Vehicle (UAV) has possibilities as the next-generation platform. In this study, we conducted a demonstration experiment to use UAV as a sea-surface base for AUV's underwater communication. We additionally verified the capability of UAV, which can land on and separate from the sea surface, as a drifting buoy to monitor underwater data. Experiment results and obtained data suggest that UAV is suitable for a research operation near the coastal region in terms of position keeping ability, performance against sway, and operation speed as a communication base. To carry out more complicated work (transportation of AUV, etc.) with UAV, new research and development such as weight reduction of AUV will be required.
\end{abstract}

Keywords: UAV; AUV; underwater communication; buoy

\section{Introduction}

Autonomous Underwater Vehicles (AUVs) have been used for oceanographic surveys because of their ability to get close to the seafloor and to obtain high-resolution seafloor images and seafloor terrain information. AUVs cannot directly acquire Global Navigation Satellite System (GNSS) signals due to the rapid attenuation of radio waves in the water. Therefore, it is necessary to deploy base stations such as ships, buoys, and autonomous vessels on the sea surface [1-4]. However, it is not easy for sea-surface systems to follow AUVs because they are drifted by disturbances such as waves and winds. Also, the surface vehicles are constrained by the sea surface, so the degree of freedom of movement and speed are low.

As described above, the next-generation navigation / communication base as a seasurface station is required to have efficient and high-speed position movement and stability. Unmanned Aerial Vehicle (UAV) is a good candidate for a sea-surface station. Especially in the coastal areas where small AUVs are used, the mobility of helicopter-type UAVs, not airplane-type UAVs, is well usable. UAVs with high moving speed (> about 50 $\mathrm{km} / \mathrm{h}$ ) and high position-keeping ability (not affected by ocean currents and perturbations) can be used for underwater exploration using such as AUVs. Additional advantage is that there is no underwater noise compared to vessels. UAVs do not generate noise underwater, making them ideal for acoustic communications.

In recent years, some types of UAVs have been developed and used for not only onshore surveying but also underwater surveying, imaging and observation [5]. Floating UAVs are also being developed but are primarily in-tended for photo and chemical surveillances in calm environments such as lakes [6]. Also, it has been developed as an observation platform with lower manufacturing cost, operating cost, and superior mobility 
[7-8]. In this study, we examined the potential use of the UAV as a 'self-propelled buoy' for ocean surveys (sea surface observation and acoustic sensing).

Figure 1 shows assuming cases in which UAVs are used as sea-surface bases for underwater explorations. Figures $1 \mathrm{a}$ and $1 \mathrm{~b}$ show that an acoustic communication sonar is equipment to UAV and can communicate with AUV. When used as a navigation / communication base for AUV, it can be divided into a case where it is made to stand by on the sea and a case where UAV lands on the sea surface. It is also possible to connect an underwater exploration device directly to UAV and carry out all surveys together with the sea-surface base using only UAVs (Figures $1 \mathrm{c}$ and $1 \mathrm{~d}$ ). Transportation of AUV is also conceivable (Figures 1e and 1f).

In this study, we conducted two demonstration experiments as in Figure 2. (1) We investigated the variation between the positioning record of an underwater sonar and the GNSS record of the positional difference between the two UAVs, and empirically verified whether they could be used for underwater communications. (2) When using a sea-surface landing $\mathrm{UAV}$, it is necessary to pay attention to the instability of communication due to sway. So, the stability against sway in the coastal area was investigated. This is because the probability of communication blackout increases, if the agitation is too large when transmitting an acoustic signal. The results of this study show the base station capability level of UAVs for AUV explorations. 


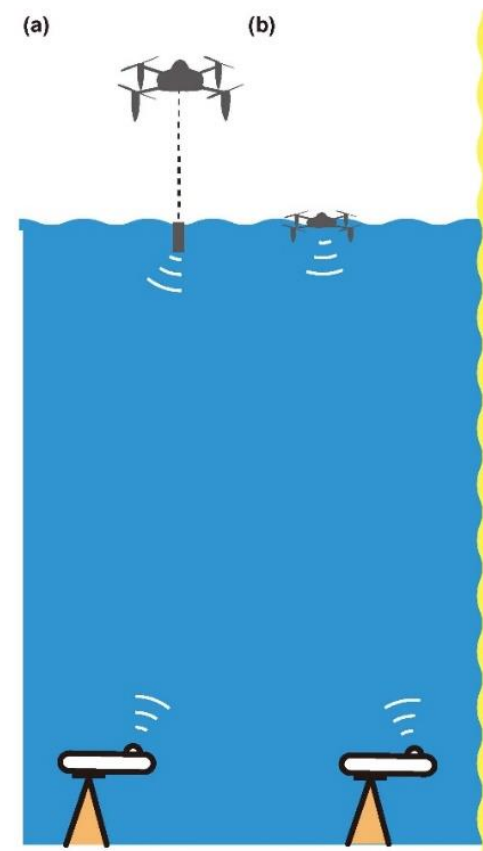

(c)

(d)

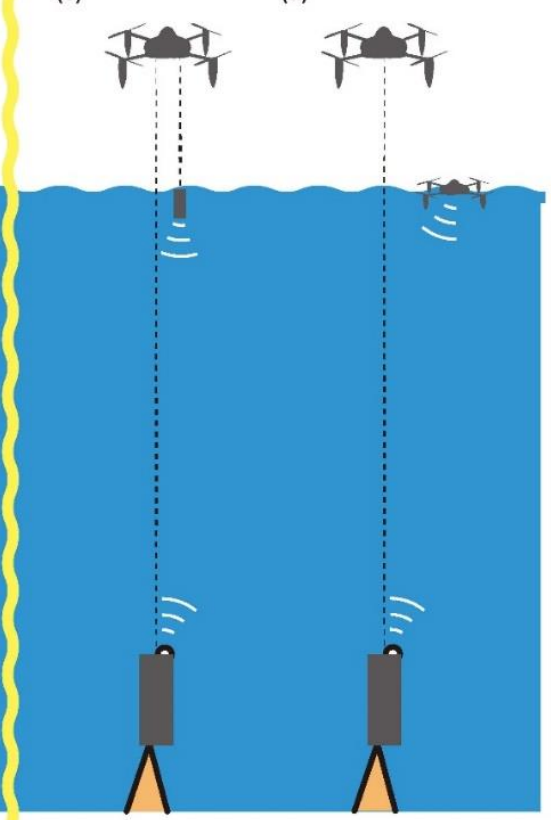

(e)

(f)
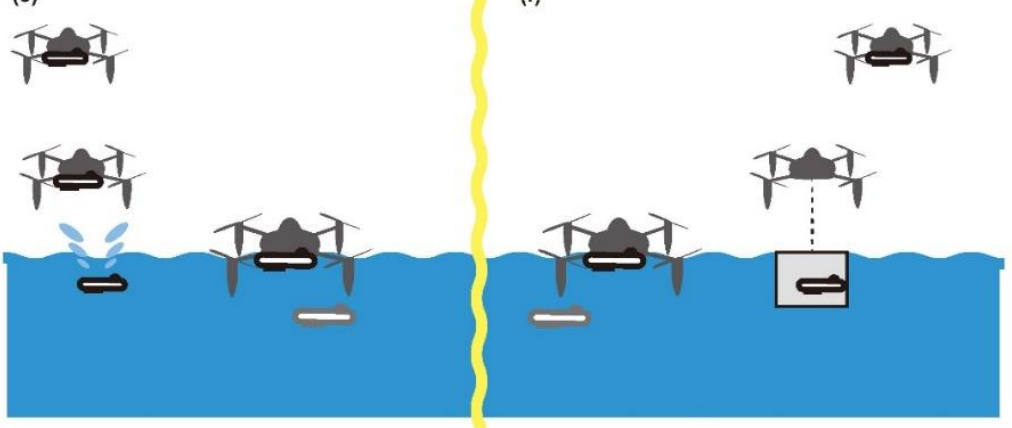

Figure 1. Conceptual schematics for underwater communication using UAV and AUV. (a) Case of hanging an acoustic base station on the sea surface with UAV during AUV survey. (b) Case of installing an acoustic base station on sea-surface UAV during AUV survey. (c) Case of hanging an underwater sonar and an acoustic base station on UAV. (d) Case of suspending an underwater sonar on UAV and installing an acoustic base station on sea-surface UAV. (e) Case for transporting AUV by UAV. (f) Case of collecting AUV with UAV.

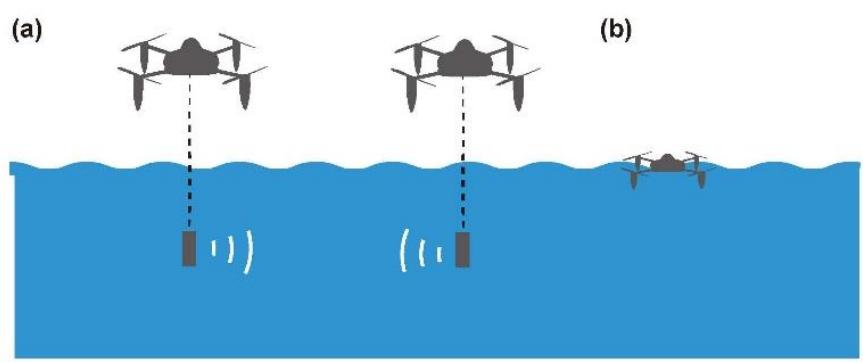

Figure 2. Settings of demonstration experiments in this study. (a) Underwater communication experiment with two UAVs for verification of stability at a distance. (b) Verification experiment of the sway for the sea-surface UAV as a buoy on the sea surface. 


\section{Materials and Methods}

Two types of UAVs were used in this study. The first one used in the experiment in subsection 3.1 is UAV that cannot land on the sea surface (Photo 1a). The UAV used here is PD6B-TypeII manufactured by PRODRONE Co., Ltd. The maximum payload of this $\mathrm{UAV}$ is about $30 \mathrm{~kg}$, and many observation instruments can be installed. A maximum flight speed of about $60 \mathrm{~km} / \mathrm{h}$ can be obtained, but a distance range of about $1 \mathrm{~km}$ is a measurement target depending on the balance between energy and communication range. The overall height of this UAV is about $55 \mathrm{~cm}$, the width is about $1.4 \mathrm{~m}$, and the weight without batteries is about $11.5 \mathrm{~kg}$. The propeller itself has a diameter of about $71 \mathrm{~cm}$.

Another one used in the experiment in subsection 3.2 is a lighter UAV that allows for takeoff and landing on the sea surface (Photos 2 and 3). The base aircraft used here is PD4AW-AQ manufactured by PRODRONE Co., Ltd. It has a float and can operate like a buoy after landing. However, the loading weight is smaller than the first UAV and can only carry up to about $4 \mathrm{~kg}$. This UAV can fly at a maximum speed of $80 \mathrm{~km} / \mathrm{h}$. The overall height of this UAV is $39 \mathrm{~cm}$, the width is $1.06 \mathrm{~m}$, and the weight without batteries is 4.6 $\mathrm{kg}$. The diameter of the propeller itself is about $43 \mathrm{~cm}$. Floats are installed below the four propellers considering a mechanism for floating. The landing operation is performed so that the float enters the sea surface almost simultaneously (almost like landing). In the case of sea surface separation, if the propellers are under the water, the aircraft cannot be separated stably. In that case, the separation operation is performed by controlling the propellers to enter the air and then to separate after a lapse of time.

These UAVs are equipped with an inertial measurement unit (IMU) that measures the motion of the aircraft for flight control purposes. In our experiment, the motion data was obtained from this IMU inside the aircraft using Wifi. It is designed so that it can be acquired at a sampling rate of $20 \mathrm{~Hz}$.

These UAVs are powered by a lithium-polymer battery (LIPO) and its flight time is limited to about 20 minutes. Stable flight is possible even when the wind speed is about $10 \mathrm{~m} / \mathrm{s}$. The data used in this study were acquired by the single-frequency GNSS mounted on the UAVs, the underwater communication device, and the IMU.

\section{Results}

\subsection{Underwater communcation experiment for two UAVs}

First, we introduce an underwater equipment used in the first experiment in Photo 1b. Underwater communication equipment includes a Lithium-ion battery (LIB; 14.8V, $18 \mathrm{Ah})$, a LIPO (5V, 10Ah), and a small CPU (UP Board UP-CHT01-0464) mounted in a water-resistant container. The acoustic positioning and communication device (Seatrac X150) was also installed next to the water-resistant container. These devices weigh less than $4 \mathrm{~kg}$. This device was lifted with a nylon rope (Photo 1c) and installed so that it stayed within a range of 1-3 $\mathrm{m}$ below sea level (Video S1).

We prepared two UAVs (the acoustic signal sending side and the receiving side) with the same equipment settings as shown in Photo $1 \mathrm{~b}$, and slowly separated the sending side and the receiving side (Photo 1d). The distance between the sonars associated with the UAVs was observed. In addition, the distance of the UAVs in the air was also observed by the GNSS. At the end of the experiment, the equipment was lifted from the sea and then the UAV landed at the base (Video S2). 
Figures $3 \mathrm{a}$ and $3 \mathrm{~b}$ show distances monitored by acoustic sensors in the first and second flights, respectively. In the second flight, the receiving-UAV flew in a farther region. Figure $3 \mathrm{c}$ also shows the differences between acoustic sensor results and the GNSS distances (A-G: acoustic sensor results - GNSS distances). In this experiment, the acoustic signal path did not pass at distances of more than $180 \mathrm{~m}$ (Figure 3b). According to the comparison with the distance of GNSS (Figure 3c), the A-G is within $\pm 3-4 \mathrm{~m}$. This A-G can be considered as approximately the horizontal distance difference between the UAV and the underwater equipment. Since the average of the A-Gs were about $-2 \mathrm{~m}$, acoustic sensors were flowed slightly outward with respect to UAVs. The $\pm 3-4 \mathrm{~m}$ variation is the sum of errors and the marine current effect. This error level is small as an underwater measurement.

Photo 1. (a) Observing a UAV that hangs an underwater communication device. Equip-

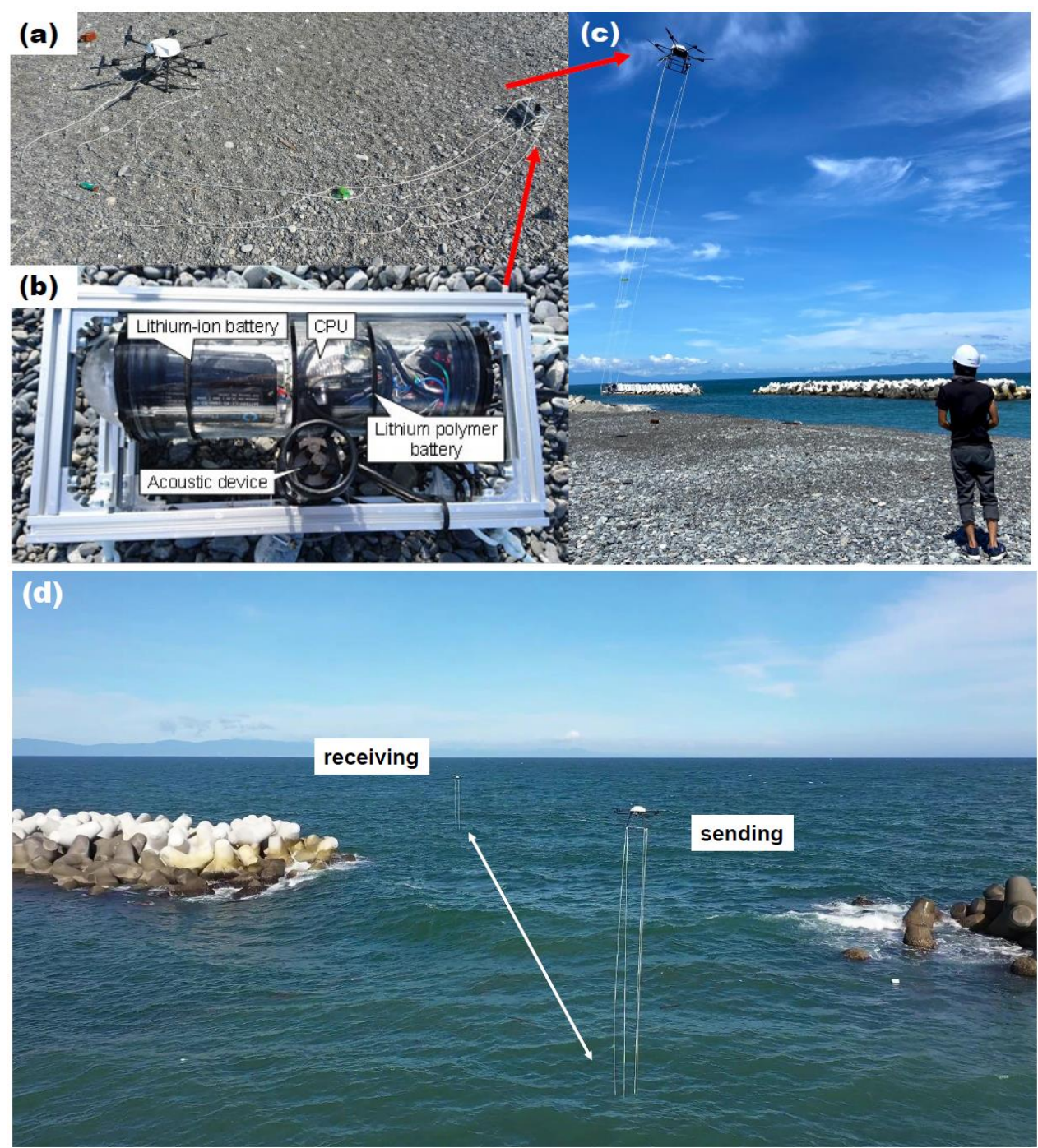

ment was suspended with nylon ropes. (b) Suspended equipment. The battery, small CPU, and acoustic device are included in one set. (c) UAV takeoff. The lower right operator controlled UAVs. (d) Receiving-UAV moved slowly away from sending-UAV. 
(a)
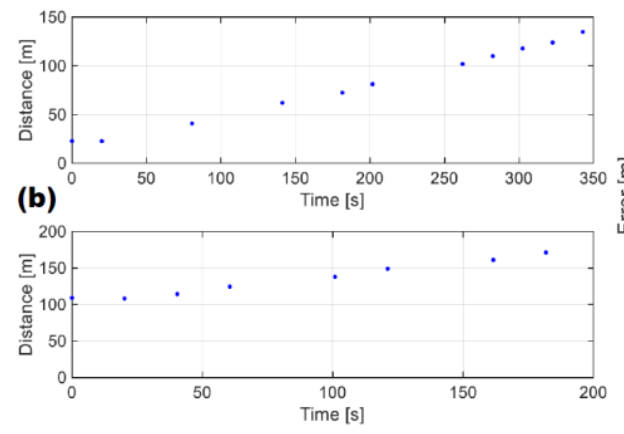

(c)

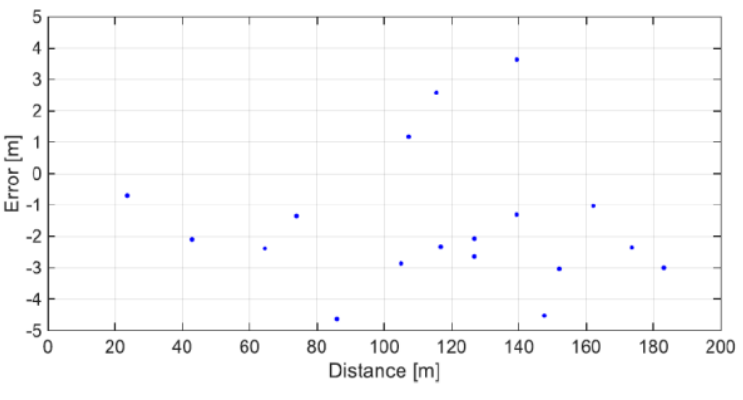

Figure 3. ( $a$ and $b$ ) Changes in the distance between two UAVs. The horizontal axis is the elapsed time, and the vertical axis is the distance monitored using acoustic devices. Results in (a) and (b) show the distances of the first and second flights, respectively. (b) The difference between the distance measured by the underwater acoustic devices and the distance of the GNSS mounted on the UAV. The horizontal axis is the GNSS distance, and the vertical axis is the distance difference (acoustic sensor results - GNSS distances).

\subsection{Additional experiment for motion data of sea-surface $U A V$}

Next, as a supplement, a verification experiment for the sway on the sea-surface landing UAV (Photo 2) was conducted. As shown in Photo 3, the UAV landed on the sea surface near the coastal area and recorded sway as a sea-surface buoy.

Figure 4 shows a part of the IMU motion data $(20 \mathrm{~Hz})$ obtained in this experiment. The UAV floated on the sea surface about 30 minutes. There was a break in the data for about $10 \mathrm{~min}$, which was thought to be due to the water leakage. The maximum motion of the UAV body was about 19 degree in the roll direction and about 18 degree in the pitch direction. While holding the sea surface, the standard deviation of motion was 4.4 degree $(1 \sigma)$ for the roll direction and 3.9 degree $(1 \sigma)$ for the pitch direction.

The sensitivity angle of the acoustic sonar Seatrac X150 used in the experiment in subsection 3.1 was about \pm 30 degrees. In this case, the sensitivity is not impaired with this degree of sway. The behavior of the UAV as a floating body on the sea surface depends on the sea surface environment due to the weather. The weather during the experiment was not a good condition with a wind speed of about $5-10 \mathrm{~m} / \mathrm{s}$, and the wave height was about $1 \mathrm{~m}$, but it can be confirmed that this UAV works as a so-called ocean measurement buoy. This UAV will work as a marine buoy with very low work costs, as no ship work occurs during loading and unloading.

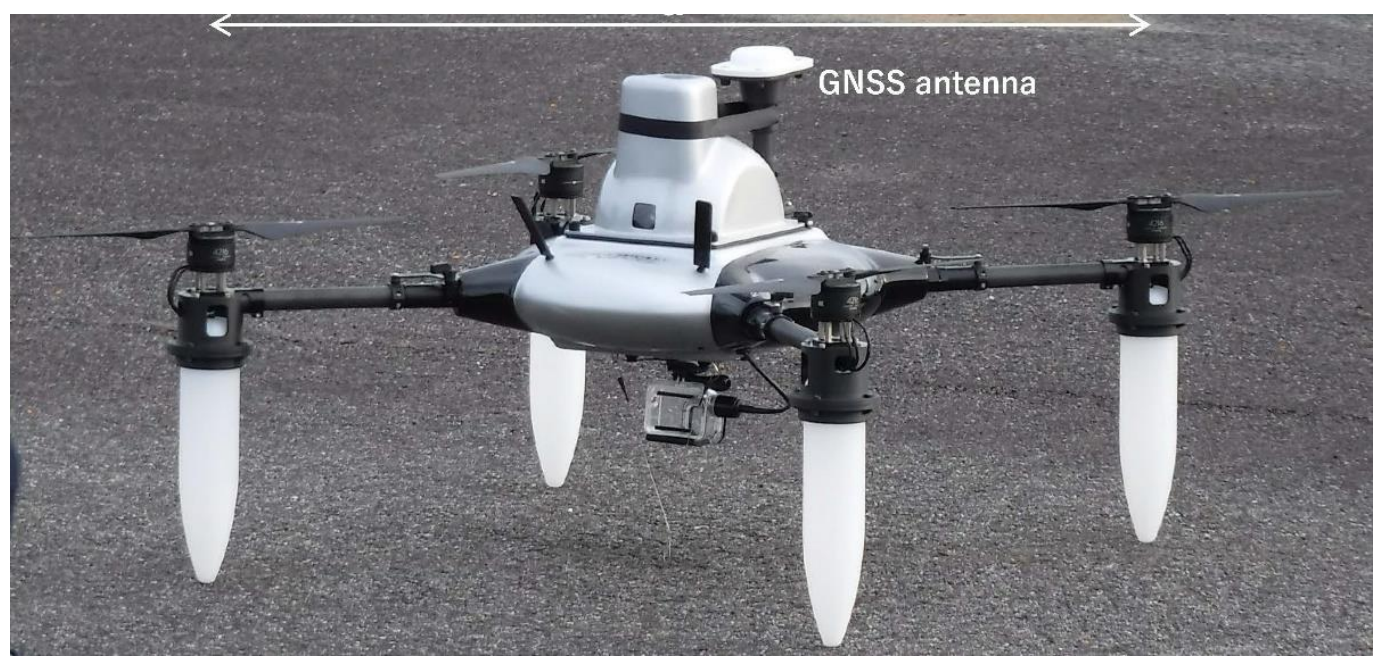

Photo 2. Sea-surface UAV. 

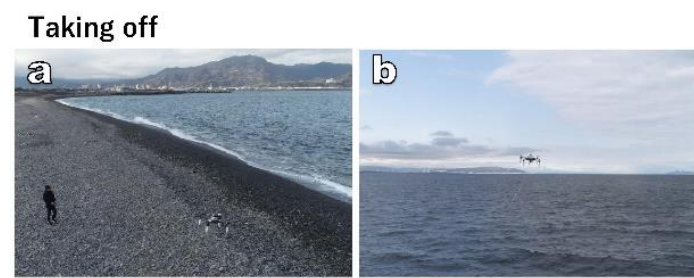

Sea surface landing
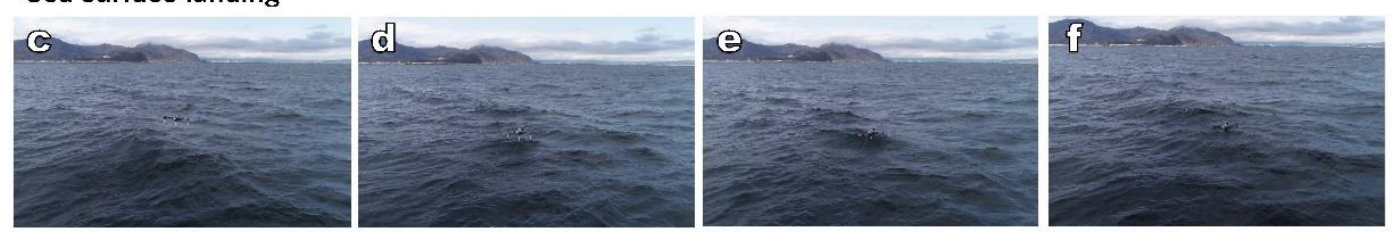

Separation from sea surface
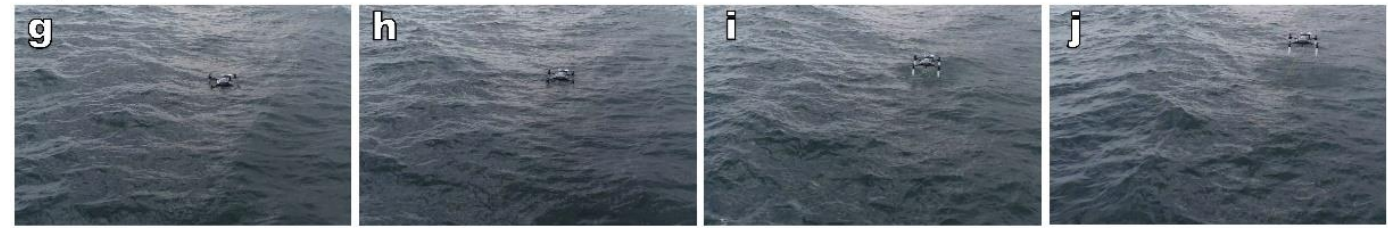

Photo 3. A snapshot of UAV operations. (a) UAV takeoff from the base. (b) UAV moving to the observation point. (c-f) A snapshot of UAV landing. (g-j) A snapshot of UAV separation. 


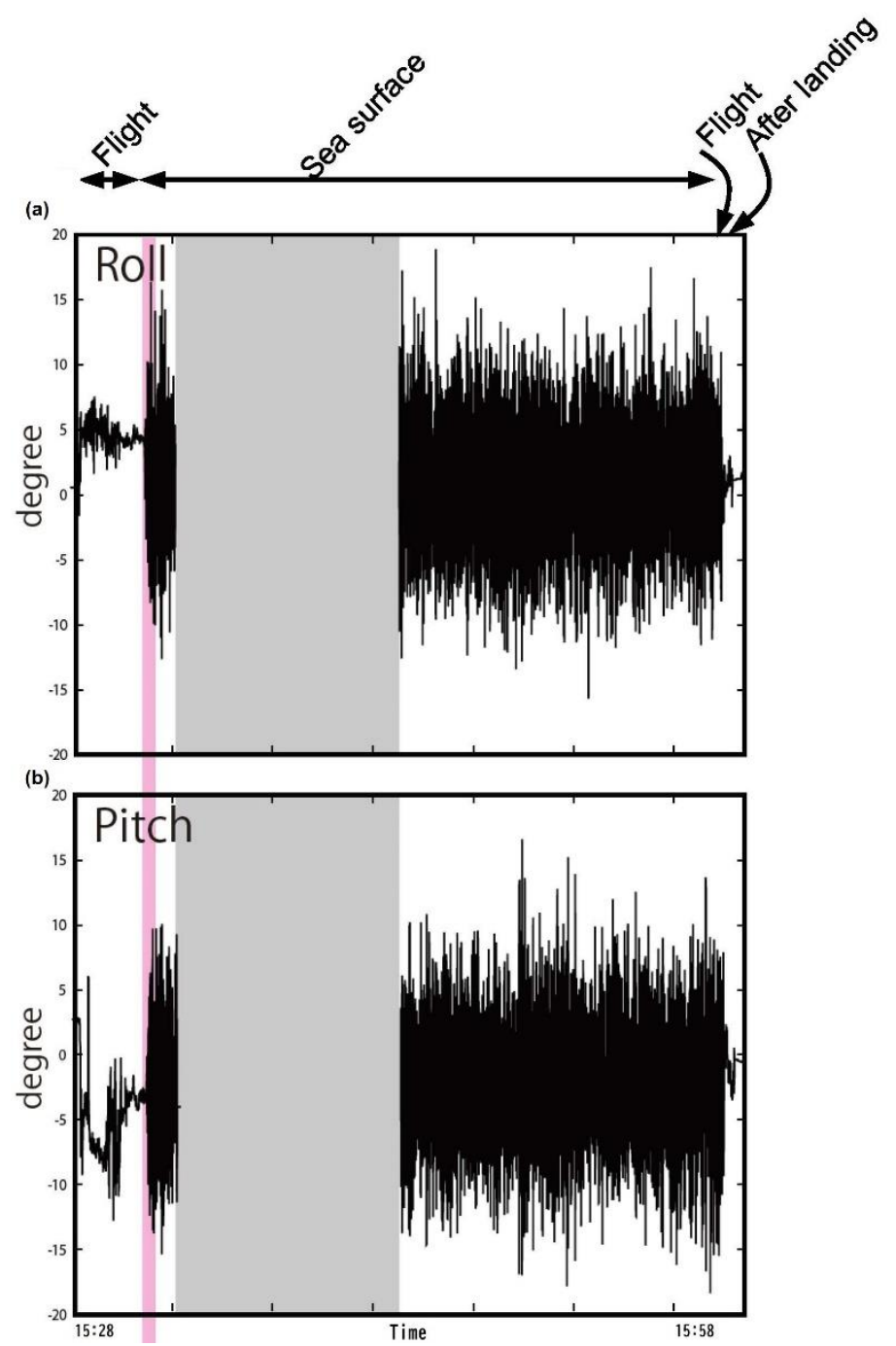

Figure 4. IMU recording of (a) the roll component and (b) the pitch component for about 30 minutes. The horizontal axis is time (JST, UTC+0900). Gray colored period indicates the period during which the measurement was missing. Red colored period is a period of landing on the sea surface.

\section{Discussion}

Table 1 shows the actual time schedule at the time of the communication experiment in subsection 3.1. It is characterized by a short flight time (2-3 minutes) to the observation point. Usually, when such an experiment is carried out using a vessel, it takes a large time cost to arrange the experiment setting and move to the observation point. UAV observation methods will reduce the cost of many observation operations.

However, in UAV experiment, there are restrictions on a flight time and an equipment weight, and continuous operation for a long time is not possible. There is a problem that the energy source of UAV depends on the LIB-like battery system. This can be solved by changing the energy source of UAV to a gasoline engine and increasing the size, but the characteristics of UAV, which is originally small and easy to operate, are lost. For solving problems, a technological innovation such as the appearance of all-solid-state batteries is required in the future.

When considering the case of transporting marine measuring equipment such as AUV by UAV (as shown in Figures 1e and 1f), the weight problem of equipment and AUV is also important. AUVs are underwater equipment and usually give a large weight because they need to be submerged. Then, many of AUV equipment are heavy, and are often 
unsuitable for transportation by UAV. The convenience of the UAV shown in this study was as a sea level base. For considering a system that leaves all operations to the UAV, new research topics for reducing the weight of the AUV should also be considered in the future.

\begin{tabular}{|r|l|}
\hline \multicolumn{1}{|l|}{$\begin{array}{l}\text { Time [hour] } \\
\text { (JST, UTC+0900) }\end{array}$} & Action \\
\hline $14: 44$ & Takeoff \\
\hline $14: 46$ & Measurement start \\
\hline $14: 52$ & Measurement end \\
\hline $14: 54$ & Landing \\
\hline & \\
\hline $16: 23$ & Takeoff \\
\hline $16: 25$ & Measurement start \\
\hline $16: 30$ & Measurement end \\
\hline $16: 32$ & Landing \\
\hline
\end{tabular}

Table 1. Actual time schedule at the time of the communication test in subsection 3.1.

\section{Conclusion}

In this study, the advantages of using UAV as a sea level base in terms of speed and maneuverability were shown and empirically verified by a demonstration experiment. The obtained data showed that UAV has no problem in terms of position keeping ability and performance against sway as a communication base. Considering a work efficiency and a measurement speed, an underwater communication technology using UAV has a potential to be a main method for underwater measurement in the coastal area up to about $1 \mathrm{~km}$ at a horizontal distance. The AUV weight is also an issue, and a new perspective has emerged in the development of underwater robots.

Supplementary Materials: Videos S1 and S2

Video S1. Observation scenery of two UAVs. The audio sensors of both machines were submerged in the sea.

Video S2. Observation scenery of two UAVs. The audio sensors were pulled up and the UAVs landed.

Author Contributions: Conceptualization, Y.Y. and T.M.; development, Y.Y. and T.M.; validation, Y.Y. and T.M.; resources, Y.Y.; writing Y.Y. and T.M.; project administration, Y.Y.; funding acquisition, Y.Y. All authors have read and agreed to the published version of the manuscript.

Funding: This research was funded by IIS selection research grant and the University of Tokyo Excellent Young Researcher project.

Data Availability Statement: The data used in this study are available from the corresponding author on reasonable request.

Acknowledgments: Shiyu Kojima, Masahito Ito, Taiju Ukai, and Naoto Sumiyoshi cooperated in the use, processing, and operation of the UAV. Taiju Ukai (Le Ciel DRONE, Co., Ltd.) also cooperated in filming and editing the movie.

Conflicts of Interest: The authors declare no conflict of interest.

\section{References}

1. Matos, A.; Almeida, R.; Cruz, N., Man portable acoustic navigation buoys. MTS/IEEE OCEANS2016-Shanghai, 2016. doi: 10.1109/OCEANSAP.2016.7485391.

2. Papadopoulos, G.; Fallon, M.F.; Leonard, J.J.; Patrikalakis, N.M., Cooperative Localization of Marine Vehicles using Nonlinear State Estimation. Intelligent Robots and System (IROS 2010), 2010. doi:10.1109/IROS.2010.5650250. 
3. Abreu, P. C.; Bayat, M.; Botelho, J.; Góis, P.; Gomes, J.; Pascoal, A.; Ribeiro, J.; Ribeiro, M.; Rufino, M.; Sebastião, L.; et al., Cooperative formation control in the scope of the EC MORPH project: Theory and experiments. MTS/IEEE OCEANS 2015-Genova, 2015. doi:10.1109/OCEANS-Genova.2015.7271697.

4. Majid, M.; Arshad, M. Design of an autonomous surface vehicle (ASV) for swarming application. IEEE/OES Autonomous Underwater Vehicles (AUV) 2016, 2016. doi:10.1109/AUV.2016.7778676.

5. Ventura, D.; Bruno, M.; Lasinio, G. J.; Belluscio, A.; Ardizzone, G. A low-cost drone based application for identifying and mapping of coastal fish nursery grounds. Estua. Coast. Shelf Sci. 2016, 171, 85-98. doi:10.1016/j.ecss.2016.01.030

6. Yao, D.; Cheng, L.; Wu, Q.; Zhang, G.; Wu, B.; He, Y. Assessment and prediction of fishery water quality using electrochemical sensor array carried by UAV. 2019 IEEE ISOEN, 18960387, 2019. doi:10.1109/ISOEN.2019.8823406

7. Goerzen, C.; Long, Z.; Mettler, B. A survey of motion planning algorithms from the perspective of autonomous UAV guidance. J. Intell. Robot. Syst. 2010, 57, 65-100. doi:10.1007/s10846-009-9383-1

8. Marek, L.; Miřijovský, J.; Tuček, P. Monitoring of the Shallow Landslide Using UAV Photogrammetry and Geodetic Mea surements. in: Lollino, G. et al. (eds) Engineering Geology for Society and Territory - Volume 2. Springer, Cham, 2015. doi:10.1007/978-3-319-09057-3_8 